\title{
The minority game: An economics perspective
}

\author{
Willemien Kets*
}

June 27, 2007

\begin{abstract}
This paper gives a critical account of the minority game literature. The minority game is a simple congestion game: players need to choose between two options, and those who have selected the option chosen by the minority win. The learning model proposed in this literature seems to differ markedly from the learning models commonly used in economics. We relate the learning model from the minority game literature to standard game-theoretic learning models, and show that in fact it shares many features with these models. However, the predictions of the learning model differ considerably from the predictions of most other learning models. We discuss the main predictions of the learning model proposed in the minority game literature, and compare these to experimental findings on congestion games.
\end{abstract}

JEL classification: C73, C90.

Keywords: Learning, congestion games, experiments.

*Address: Tilburg University, P.O. Box 90153, 5000 LE Tilburg, The Netherlands. E-mail: w.kets@uvt.nl. Tel: +31-13-4662478. Fax: +31-13-4663280. I am indebted to Ginestra Bianconi, George Ehrhardt, Doyne Farmer, Matteo Marsili, Esteban Moro, Jan Potters, Dolf Talman, and Mark Voorneveld for inspiring discussions and helpful comments and suggestions. In addition, I would like to thank Esteban Moro for his kind permission for reproducing some of the figures from Moro (2003). All remaining errors are of course my own. 


\section{Introduction}

Congestion games are ubiquitous in economics. In a congestion game (Rosenthal, 1973), players use several facilities from a common pool. The costs or benefits that a player derives from a facility depends on the number of users of that facility. A congestion game is therefore a natural game to model scarcity of common resources. Examples of such systems include vehicular traffic (Nagel et al., 1997), packet traffic in networks (Huberman and Lukose, 1997), and ecologies of foraging animals (DeAngelis and Gross, 1992). Similar coordination problems are encountered in market entry games (Selten and Güth, 1982).

Congestion games are also interesting from a theoretical point of view. In congestion games, players need to coordinate to differentiate. This seems to be more difficult than coordinating on the same action, as any commonality of expectations is broken up. For instance, when commuters have to choose between two roads $A$ and $B$ and all believe that the others will choose road $A$, nobody will choose that road, invalidating beliefs. The sorting of players predicted in the pure-strategy Nash equilibria of such games violates the common belief that in symmetric games, all rational players will evaluate the situation identically, and hence, make the same choices in similar situations (see Harsanyi and Selten, 1988, p. 73). Moreover, in congestion games, players may obtain asymmetric payoffs in equilibrium which may complicate attainment of equilibrium, as coordination cannot be achieved through tacit coordination based on historical precedent (cf. Meyer et al., 1992). Finally, congestion games often have many equilibria, so that players also face the difficulty of coordinating on the same equilibrium.

Nevertheless, the theory of learning in games provides sharp predictions on players' behavior in congestion games. As congestion games belong to the class of potential games (Monderer and Shapley, 1996b), all results that have been derived for potential games apply to the class of congestion games 1 Experimental evidence, however, is not always in line with these predictions. Though several experimental studies have shown that players are remarkably successful at learning to coordinate in congestion games, 2 regularities on

\footnotetext{
${ }^{1}$ See e.g. Hofbauer and Hopkins (2005), Hofbauer and Sandholm (2002), Monderer and Shapley (1996b), and Sandholm (2001, 2007). Kets and Voorneveld (2007) study the convergence of play under different learning processes in the minority game.

${ }^{2}$ For instance, interacting players rapidly achieve a "magical" degree of tacit coordination in market entry games, which is accounted for on the aggregate level by the Nash equilibrium solution (Kahneman, 1988; Rapoport, 1995; Sundali et al., 1995; Erev and Rapoport, 1998; Rapoport et al., 1998, 2000). See e.g. Meyer et al. (1992) and Selten et al. (2007) for similar results on related games.
} 
the aggregate level generally conceal non-equilibrium behavior at the individual level. Even though aggregate play is close to the Nash equilibrium, individual players generally do not play equilibrium strategies 3 Moreover, providing players with more information does not always lead to better outcomes 4

These experimental findings are hard to explain with standard learning models. This paper discusses the literature on the minority game, a simple congestion game based on the El Farol bar problem of Arthur (1994). Players have to choose between two alternatives. Only those who have chosen the minority side get a positive payoff. The minority game literature proposes a learning model that is able to account for many of the experimental findings listed above. We relate this learning model to the standard learning models in economics, and compare its predictions to experimental results on congestion games. The contribution of the current paper is that it relates the literature on the minority game, which has been largely developed in physics, to the literature on learning in game theory and to the literature in experimental economics on congestion games 5

The outline of this paper is as follows. In Section 2, we introduce the minority game and discuss its equilibria. The learning model proposed in the minority game literature is discussed in Section 3. In Section 4, we discuss the main predictions from the learning model. These predictions are compared to experimental results on congestion games in Section 5. Section 6 concludes.

\section{The stage game}

The minority game is a game in which an odd number of players have to choose between two actions; for instance, players either go to a bar or stay home, either buy or sell an asset, etcetera. Players want to distinguish themselves from the crowd: their aim is to take a different action than the majority of players.

\footnotetext{
${ }^{3}$ See e.g. Meyer et al. (1992), Erev and Rapoport (1998), Selten et al. (2007), Bottazzi and Devetag (2004).

${ }^{4}$ For instance, in their experiments on market entry games, Erev and Rapoport (1998) find that providing players with information on other players' actions may actually lead to lower average payoffs.

${ }^{5}$ We have no intention of giving a comprehensive survey of the minority game literature, as an enormous amount of work on the minority game has been done. For an extensive collection of papers on the minority game, see http://www.unifr.ch/econophysics/minority/. See Moro (2003); Challet et al. (2004) or Coolen (2005) for an introduction to the field. Papers in economics on the minority game include Bottazzi and Devetag (2007), Chmura and Pitz (2004), and Renault et al. (2005). Blonski (1999) and Kọima and Takahashi (2004) study learning in games very similar to the minority game.
} 
Following the notation of Tercieux and Voorneveld (2005), we denote the set of players by $\mathcal{N}=\{1, \ldots, 2 k+1\}$, with $k \in \mathbb{N}$. Each player $i \in \mathcal{N}$ has a set of pure strategies $A_{i}=\{-1,+1\}$ : agents have to choose between two options. The set of mixed strategies of player $i$ is denoted by $\Delta\left(A_{i}\right)$. We denote a mixed strategy profile by $\alpha \in \times_{i \in \mathcal{N}} \Delta\left(A_{i}\right)$, and we use the standard notation $\alpha_{-i} \in \times_{j \in \mathcal{N} \backslash\{i\}} \Delta\left(A_{j}\right)$ to denote a strategy profile of players other than $i \in \mathcal{N}$. With each action $a \in\{-1,+1\}$, a function

$$
f_{a}:\{1, \ldots, 2 k+1\} \rightarrow \mathbb{R}
$$

can be associated which indicates for each $n \in\{1, \ldots, 2 k+1\}$ the payoffs to a player choosing $a$ when the total number of players choosing $a$ equals $n$. The von NeumannMorgenstern utility function of a player is then given by

$$
u_{i}(a)=f_{a_{i}}\left(\left|\left\{j \in \mathcal{N}: a_{j}=a_{i}\right\}\right|\right),
$$

where $a \in \times_{j \in \mathcal{N}} A_{j}$. Payoffs are extended to mixed strategies in the usual way.

The function $f_{a}(\cdot), a \in\{-1,+1\}$ can have several forms. It is commonly assumed that congestion is costly:

[Mon] $\quad f_{-1}$ and $f_{+1}$ are strictly decreasing functions,

and that the congestion effect is the same across alternatives:

$[\mathrm{Sym}] \quad f_{-1}=f_{+1}$.

A commonly used form is $f_{-1}(n)=f_{+1}(n)=1$ if $n \in\{1, \ldots, k\}$ and 0 otherwise (Challet and Zhang, 1997). Alternatively, one could define payoffs in terms of the aggregate action $\sum_{i \in \mathcal{N}} a_{i}$ for a given action profile $a=\left(a_{i}\right)_{i \in \mathcal{N}}$, with $a_{i} \in\{-1,+1\}$ for all $i$. Let $g$ be a function on $\mathbb{R}$ such that $g(-x)=-g(x)$ for all $x \in \mathbb{R}$ and $g(x)>0$ for $x>0$. A player $i \in \mathcal{N}$ is then assigned the payoff

$$
u_{i}(a)=-a_{i} g\left(\sum_{j \in \mathcal{N}} a_{j}\right) .
$$

In our notation:

$$
f_{-1}(n)=f_{+1}(n)=g(2(k-n)+1) .
$$

Common choices include

$$
g(x)=x /(2 k+1)
$$


and

$$
g(x)=\operatorname{sign}(x)
$$

Most of the predictions of the learning model are not affected qualitatively by the precise choice of payoff function, given that it satisfies [Mon] and [Sym] (Li et al., 2000). Notice that the minority game is a congestion game (Rosenthal, 1973) and hence a finite exact potential game (Monderer and Shaplev, 1996b).

To analyze the game's Nash equilibria, we introduce some more notation. A player who uses a mixed strategy that puts positive probability on both pure strategies is referred to as a mixer. A player that puts full probability mass on the alternative -1 is called a $(-1)$-player; similarly, a player that puts full probability mass on the alternative +1 is called a $(+1)$-player.

The stage game has a large number of Nash equilibria. Tercieux and Voorneveld (2005) show that a pure strategy profile is a Nash equilibrium if and only if one of the alternatives -1 or +1 is chosen by exactly $k$ of the $2 k+1$ players. Note that these Nash equilibria are not strict, as a player that is in the majority is indifferent between sticking to his choice or switching actions, as his deviation would shift the majority. There are $2\left(\begin{array}{c}2 k+1 \\ k\end{array}\right)$ of such asymmetric pure-strategy Nash equilibria.

Kets and Voorneveld (2007) characterize the game's mixed-strategy Nash equilibria. It can be shown that in any Nash equilibrium with at least one mixer, all mixers use the same mixed strategy. Moreover, player labels are irrelevant by [Sym] (if $\alpha$ is a Nash equilibrium, so is every permutation of $\alpha$ ). Together, these facts imply that a Nash equilibrium with at least one mixer can be summarized by its type $(\ell, r, \lambda)$, where $\ell, r \in\{0,1, \ldots, 2 k+1\}$ denote the number of players choosing pure strategy -1 or +1 , respectively, and $\lambda \in(0,1)$ the probability with which the remaining $z(\ell, r, \lambda):=(2 k+1)-(\ell+r)>0$ mixers choose -1 . Let $v_{-1}(\ell, r, \lambda)$ denote the expected payoff to a player choosing $-1 ; v_{+1}(\ell, r, \lambda)$ is defined similarly. Then, a strategy profile of type $(\ell, r, \lambda)$ is a Nash equilibrium if and only if

$$
v_{-1}(\ell+1, r, \lambda)=v_{+1}(\ell, r+1, \lambda)
$$

i.e., the expected payoffs to a mixer of playing the pure strategy $a=-1$ are equal to the expected payoffs of the pure strategy $a=+1$. It can be shown that there exist Nash equilibria with exactly one mixer. These equilibria are of type $(k, k, \lambda)$ with arbitrary $\lambda \in(0,1)$, i.e., the mixer uses an arbitrary mixed strategy, whereas the remaining $2 k$ players are spread evenly over the two pure strategies. In addition, there are Nash equilibria with more than one mixer. For $\ell, r \in\{0,1, \ldots, 2 k+1\}$ such that $\ell+r \leq 2 k-1$, there is a Nash 
equilibrium of type $(\ell, r, \lambda)$ if and only if $\max \{\ell, r\}<k$. The corresponding probability $\lambda \in(0,1)$ solves (2.4), and can be shown to be unique. It follows from these results that there is a unique symmetric mixed-strategy Nash equilibrium. In this equilibrium, each player chooses each option with probability $\frac{1}{2}$. The expected number of players choosing each option is then $k+\frac{1}{2}$.

\section{Learning in the minority game}

Players in the minority game face both a coordination problem and an incentive problem. The coordination problem is not easy to solve. As the equilibria in pure strategies cannot be Pareto-ranked or ordered in terms of risk-dominance, no particular pure-strategy Nash equilibrium can be singled out as being most salient (Schelling, 1960). Hence, without pre-play communication, players do not have enough information to implement a pure-strategy Nash equilibrium (cf. Menezes and Pitchford, 2006). While players could use common knowledge of rationality and symmetry to deduce and select the symmetric mixed-strategy Nash equilibrium (cf. Ochs, 1990; Meyer et al., 1992), this may raise an incentive problem, as players can earn a higher payoff than in the symmetric mixed-strategy Nash equilibrium if they manage to outsmart the other players. Hence, players may try to find patterns in the play of others when the game is played repeatedly (cf. Arthur, 1994; Meyer et al., 1992). The learning model proposed in the minority game literature provides a way of formalizing this notion. In this section, we first introduce the model, and then discuss its assumptions, relating the learning model to other learning models in the literature.

\subsection{Model}

The stage game is played repeatedly. After each round of play $t$ of the stage game, the players are informed of the aggregate action $A(t):=\sum_{i=1}^{2 k+1} a_{i}(t)$, where $a_{i}(t) \in\{-1,+1\}$ is the action taken by player $i$ in round $t$. Furthermore, it is assumed that players only retain the sequence of the last $m$ winning groups $-\operatorname{sign}[A(t)]$, where $m \in \mathbb{N}$. Hence, in round $t$, players observe the $m$ most recent outcomes $h_{m}(t)=(-\operatorname{sign}[A(\tau)])_{\tau \in\{t-m, t-m+1, \ldots, t-1\}}$.

A response mode $s$ assigns to each information set $h_{m} \in \mathcal{H}_{m}=\left\{\left(x_{k}\right)_{k=1, \ldots, m} \mid x_{k} \in\right.$ $\{-1,+1\}\}$ an action $a \in\{-1,+1\}$. That is, a response mode $s$ prescribes which action $s\left(h_{m}(t)\right) \in\{-1,+1\}$ to take, for a given history of play $h_{m}(t)$ at time $t$. There are $2^{2^{m}}$ different response modes: there are $2^{m}$ possible signals $h_{m}$ of length $m$, and for each signal, 


\begin{tabular}{|c|c|c|c|c|c|c|}
\hline \multicolumn{3}{|c|}{ History } & \multicolumn{4}{|c|}{ Action } \\
\hline & $h_{m}$ & & $s_{i, 1}$ & $s_{i, 2}$ & $s_{i, 3}$ & $s_{i, 4}$ \\
\hline-1 & -1 & -1 & +1 & -1 & -1 & +1 \\
\hline-1 & -1 & +1 & -1 & -1 & +1 & -1 \\
\hline-1 & +1 & -1 & +1 & -1 & -1 & +1 \\
\hline-1 & +1 & +1 & -1 & +1 & -1 & +1 \\
\hline+1 & -1 & -1 & +1 & +1 & +1 & +1 \\
\hline+1 & -1 & +1 & -1 & -1 & +1 & +1 \\
\hline+1 & +1 & -1 & -1 & -1 & -1 & +1 \\
\hline+1 & +1 & +1 & -1 & +1 & -1 & +1 \\
\hline
\end{tabular}

Table 1: An example of a subset of response modes with $m=3$ and $n_{S}=4$ for some player $i \in \mathcal{N}$.

there are two possible actions. For memory length $m$, denote the set of all response modes by $\mathcal{S}^{(m)}$. An important assumption in the minority game learning model is that each player $i \in \mathcal{N}$ is endowed with a subset $S_{i}$ of all possible response modes, with for each $i \in \mathcal{N}$ the response modes in $S_{i}$ drawn uniformly at random from $\mathcal{S}^{(m)}$, independently across players. Results are then obtained by averaging over all possible assignments of response modes. This endowment is fixed for each player, and all players are endowed with the same number $n_{S} \geq 2$ of response modes. An example of such a subset of response modes for $n_{S}=4$ and $m=3$ is given in Table 1 .

When faced with a history $h_{m}(t)$, an player has to choose which of his $n_{S}$ response modes to use in the next round. Each player $i$ keeps a virtual score $p_{i, \ell}(t)$ for each response mode $s_{i, \ell} \in S_{i}$ that reflects that response mode's past performance. The virtual score of each response mode is updated after each round, regardless of whether the response mode has been used or not. When a response mode would have correctly predicted the winning side, its virtual score is increased with the payoffs it would have earned, otherwise it is decreased with the same amount. This means that players do not take the effect of their action on the aggregate outcome $A(t)$ into account. In determining the virtual score of a response mode, players only consider whether this response mode would have predicted the actual outcome correctly, neglecting the question whether playing this response mode would have affected the outcome.

Example 3.1. Suppose that the payoffs are of the form (2.3). Then, the updating rule is:

$$
p_{i, \ell}(t+1)=p_{i, \ell}(t)-s_{i, \ell}\left(h_{m}(t)\right) \cdot \frac{A(t)}{2 k+1}
$$


where $\ell \in\left\{1, \ldots, n_{S}\right\}$. Suppose that in some round $t$, player $i$ has chosen action $a_{i}(t)=-1$, and that the total number of players choosing action $a=-1$ is $k+1$, i.e., -1 is the majority action. Then the virtual score of all response modes prescribing $a=-1$ would be decreased by $(k+1)-k=1$, while the virtual scores of all other response modes would be increased by 1 . However, if player $i$ would have played one of those response modes, the number of players choosing $a=+1$ would have been $k+1$, and +1 would have been the majority action.

The probability that player $i \in \mathcal{N}$ chooses the response mode $s_{i, \ell} \in S_{i}$ in the next round is given by the well-known logit choice rule:

$$
\operatorname{Prob}\left\{s_{i}(t)=s_{i, \ell}\right\}=\frac{e^{\beta \cdot p_{i, \ell}(t)}}{\sum_{j} e^{\beta \cdot p_{i, j}(t)}} .
$$

The parameter $\beta$ can be interpreted as the sensitivity of choice to marginal information. In the limiting case $\beta \rightarrow \infty$, play becomes fully deterministic in the sense that players choose the response mode with the highest virtual score. Allowing for $\beta<\infty$ adds noise at

the individual level as well as it introduces additional heterogeneity (Cavagna et al., 1999). When $\beta \rightarrow \infty$, all players endowed with a certain response mode keep the same virtual score for that response mode. By contrast, for finite $\beta$, players differ in their ranking of response modes, as their endowment of response modes determines the denominator of Equation (3.1). Perhaps surprisingly, this added heterogeneity and noise actually improves collective performance, as discussed in Section 4.1.

Actions, outcomes and performance are thus linked by a complex feedback system, as illustrated in Figure 3.1. Players observe the recent outcomes, and choose a response mode with a probability depending on the number of virtual points that response mode collected, resulting in an action $a \in\{-1,+1\}$. The actions of all players determine the winning side through the minority rule; this information is then fed back to the players and adds to the sequence of outcomes.

\subsection{Discussion}

In this section, we discuss two of the most important assumptions of the learning model in the minority game model: the assumption that all players are endowed with a random subset of response modes and the assumption that players update the virtual scores of response modes not used, without taking into account the effect of that response mode on the game's outcome. Although the learning model of the minority game literature 


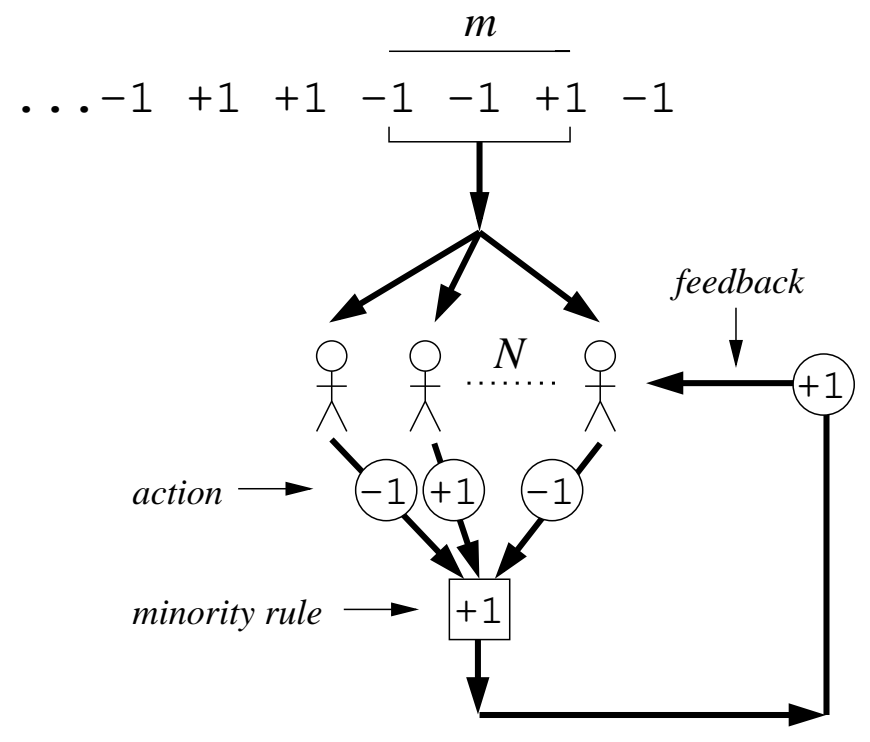

Figure 3.1: A schematic overview of the minority game learning model. Figure taken from Moro (2003).

seems to depart markedly from the standard evolutionary and learning models used in economics, we argue here that in fact the learning model combines different aspects of several game-theoretic models to provide a realistic model of player behavior in congestion games.

\subsubsection{Response modes and heterogeneity}

In the learning model proposed in the minority game literature, players base their action on the recent past, trying to discern patterns in their opponents' behavior, as in Arthur (1994). Arthur proposes that players condition their decision to go to a bar on attendance levels in the previous weeks. He employs the terms "predictor" or "hypothesis" rather than response mode: if the bar has been crowded for the last three weeks, I expect it to be crowded next week also. These mental models are mapped into actions: if I expect the bar to be crowded, I will not go.

The response modes in the minority game learning model are a concise way of modelling this notion. An important question, however, is which response modes need to be included in the model. There are two possible avenues. Firstly, one could simply incorporate all possible response modes. However, if all possible response modes are included in the learning model, the strategy space becomes huge already for very simple games. Many 
different response modes are conceivable in a simple game such as the minority game, as illustrated by the list of examples in Arthur (1994).

A second possibility is to include only a selection of possible response modes. In that case, one could either make a selection based on behavioral assumptions, or let the subset of response modes be determined randomly. In the first case, a natural choice is to include response modes that reflect beliefs about other players' actions, based on recent outcomes. The first approach is commonly taken in the economics literature (e.g. Erev and Rapoport, 1998; Selten et al., 2007), while the minority game learning model takes the second avenue. When players' response modes are drawn uniformly at random from the set of all possible response modes, there are no restrictions on the types of response modes that players use.

At first sight, this may seem to be a weak point of the model, as response modes do not need to have a sensible interpretation in the minority game learning model. However, it can be shown that regardless which response modes players are endowed with, players will self-organize into groups that use different response modes in such a way that their actions cancel out (see Challet and Zhang (1998); Hart et al. (2001); see also Section 4.3). Hence, the minority game learning model provides a possible explanation for the simultaneous evolution of behavioral rules (e.g. "switch roads if the road was crowded in the previous period") and their antagonists ("stay at the same road if the road was crowded in the previous period") often observed in congestion game experiments (e.g. Selten et al., 2007) through the structure of the game and players' heterogeneity. The strong point of the minority game learning model is exactly that no assumptions regarding response modes are needed. In games such as the minority game, whether a response mode is reasonable only depends on the response modes used by others 6 Conversely, any response mode, whether it has a sensible interpretation or not, will work if opponents use response modes that recommend them to take the opposite action (see Section 4.3).

Note that the minority game differs in this respect from games such as the p-beauty contest (Keynes, 1936, p. 156) 7. Both in the p-beauty contest and the minority game,

\footnotetext{
${ }^{6}$ For instance, Selten et al. (2007) reports that some subjects use a "direct" response mode in his experiments on route-choice games, while other subjects use a "contrarian" response mode. Subject who use the former response mode will switch roads if they experienced congestion in the last period, while subjects using the contrarian response mode stick with their choice, as they expect other subjects to switch. The important point to note is that the direct response mode is only sensible if there are players who use the contrarian response mode and vice versa.

${ }^{7}$ In the p-beauty contest, players have to choose a number in a certain interval. Players have to guess what the average choice will be; the player that picks the number that is closest to some fraction $\varphi<1$ of the average choice will win. Suppose players have to choose a number between 0 and 100 , and will win
} 
players base their actions on their beliefs about other players' actions, who in turn base their actions on ..., etcetera. While in the p-beauty contest, this recursion of actions and beliefs ends at a well-defined limit point, the Nash equilibrium action, there is no such limit point in the minority game. This means that there is no action in the minority game that is optimal a priori, as in the p-beauty contest: if all think that $a=-1$ will be the minority choice, then all will choose that action 8 In such a case, agnosticism on the type of response modes that players use may well provide a more realistic model of players' reasoning processes than the more restrictive assumptions employed in different learning models. This offers an elegant solution to the dilemma signalled by Erev and Roth (1998, p. 873) that it is virtually impossible to include all possible behavioral rules, but that selection of specific rules bears the risk of "parameter fitting in a model with an enormous number of parameters". In the minority game learning model, no response mode is ruled out on a priori grounds, while sensible behavioral rules evolve naturally, as the only criterion for a behavioral rule to be sensible in the minority game is that there are other players who follow a "contrarian" behavioral rule.

However, this approach raises some questions. Firstly, one may ask why it is assumed that players are heterogeneous in their endowment of response modes. Perhaps more importantly, one could ask why players only consider a fixed number $n_{S}$ of response modes. Indeed, individual players have an incentive to increase the number of response modes they use, as that gives them an advantage over other players (Marsili et al., 2000). However, these assumptions are not uncommon in game-theoretic models of learning and bounded rationality 9 Possible justifications for such assumptions include that each player has different experiences prior to playing the minority game and therefore deems different response modes more reasonable than others (cf. Aumann, 1997; Fudenberg and Levine, 1998, p.4, and references therein), and that boundedly rational player may prefer to just consider a subset of response modes that have worked well in the past, rather than considering all $2^{2^{m}}$ response modes (cf. Ellison and Fudenberg, 1993) 10

with their choice is closest to $\varphi=2 / 3$ of the average choice. Then nobody will choose a number higher than $\frac{2}{3} \cdot 100$, so nobody should pick a number higher than $\frac{2}{3} \cdot \frac{2}{3} \cdot 100$, and so on. When players are rational and there is common knowledge of rationality, the equilibrium choice is 0 .

${ }^{8}$ Also see Camerer and Fehr (2006). Camerer and Fehr explain behavior in congestion games and the p-beauty contest using the cognitive hierarchy approach (Camerer et al., 2004; Stahl and Wilson, 1995).

${ }^{9}$ For instance, in cognitive hierarchy models (Camerer and Ho, 1999; Stahl and Wilson, 1995), it is assumed that each player is of some exogenously specified type; players of different types use different strategies. Another example is the replicator dynamic (e.g. Weibull, 1995) in which players are "programmed" to play a given strategy.

${ }^{10}$ The precise value of $n_{S}$ is irrelevant. The qualitative behavior of the model is not affected by the 


\subsubsection{The law of simulated effect and boundedly rational players}

Which response mode players choose from the set of response modes they are endowed with, is determined by the virtual score of each response mode. The learning process proposed in the minority game literature is closely related to the reinforcement learning model of Roth and Erev (1995) and Erev and Roth (1998). The main difference between the basic reinforcement learning model of Roth and Erev and the learning model of the minority game literature lies in the updating of the score of strategies or response modes not played. In the basic reinforcement learning model, the scores of these strategies are not updated, while in the minority game learning model, the scores of all response modes are updated in every period, as in hypothetical reinforcement learning or stochastic fictitious play (Fudenberg and Levine, 1998). The assumption that players also consider the payoffs to strategies or response modes not played seems to be reasonable. Camerer and Ho (1999) argue on the basis of theoretical arguments as well as on the basis of experimental results that players obey not only the "law of actual effect", but also the "law of simulated effect", meaning that in reinforcement, not only payoffs from strategies that are actually used count, but also foregone payoffs from strategies not played.

However, for players to play according to the act of simulated effect, they need more information than for standard reinforcement learning 11 In general, to play according to fictitious play, players need to know the payoff rule as well as the actions of their opponents in addition to their own payoff. Even in a game such as the minority game, where the players only need to know the aggregate choice of other players (and not their individual choices), calculating foregone payoffs of strategies not used may be too hard for players that are boundedly rational. In the minority game learning model, players' bounded rationality is reconciled with the law of simulated effect by assuming that players do not take the effect of their own action on the global outcome into account. In that way, players can account for foregone payoffs of response modes not used, without having to do complicated calculations.

At first sight, one may think that for a large number of players, it does not matter whether players account for their own impact. However, due to the minority rule, there remains a systematic bias in the rewarding of response modes, even if the number of players goes to infinity. The reason is that the virtual score of a response mode that is currently played is systematically lower than that of the response modes that are not used. These

choice of $n_{S}$, as long as there is some heterogeneity among players (Challet et al., 2004).

${ }^{11}$ Recall that players only need to know their own payoff to play according to the standard reinforcement learning model of Roth and Erev (1995). 
latter response modes get a point if they prescribe the current minority side, even if they would have tipped the minority to the other side if they would have been played, so that they would have guessed wrong in reality (cf. Example 3.1). As the response mode that is actually played does not have this advantage, the response modes that are not played are systematically favored and hence results depend on whether players take the effect of their action on the aggregate outcome into account (Marsili et al., 2000; Marsili and Challet, 2001).

The minority game learning model thus combines features from several learning models in the literature on learning in games. However, the minority game learning model makes distinctly different predictions than game-theoretical learning models. To these predictions we now turn.

\section{Predictions of the learning model}

In this section, we discuss the main predictions on the minority game learning model. In the first two sections, we characterize the behavior of the model in terms of social efficiency and informational efficiency, and show that the two are inherently linked in the minority game learning model. In Section 4.3, we show how different response modes may evolve, and discuss the implications for efficiency.

\subsection{Volatility and attendance}

Typically, dust never settles down in the minority game learning model: the aggregate attendance $A(t):=\sum_{i \in \mathcal{N}} a_{i}(t)$ as a function of round number $t$ keeps fluctuating, as can be seen in Figure 4.1. As the game is symmetric, the time average of $A(t)$ will be 0 in the steady state, as borne out by simulations (see e.g. Challet and Zhang, 1997, 1998; Johnson et al., 1998; Manuca et al., 2000). More interesting is the behavior of the variance $\sigma^{2}:=\left\langle A^{2}\right\rangle$, where $\langle\cdot\rangle$ denotes the (time) average of a quantity. The variance, or volatility, is a measure of the degree of efficiency achieved in a population. The higher the variance, the larger the aggregate welfare loss: large fluctuations around the time average $\langle A\rangle=0$ imply that the size of the minority is only small. When payoffs are linear in $A(t)$, this is easy to see: in that case, total payoffs are proportional to $-\sum_{i \in \mathcal{N}} a_{i} A(t)=-(A(t))^{2}$.

It has been found that $\sigma^{2}$ is only a function of $\alpha:=2^{m} /(2 k+1)$ for a given value of $n_{S}$, where we recall that $n_{S}$ is the number of response modes of each player (Savit et al., 1999). Figure 4.2 shows the volatility as a function of $\alpha$. As can be seen in the figure, 


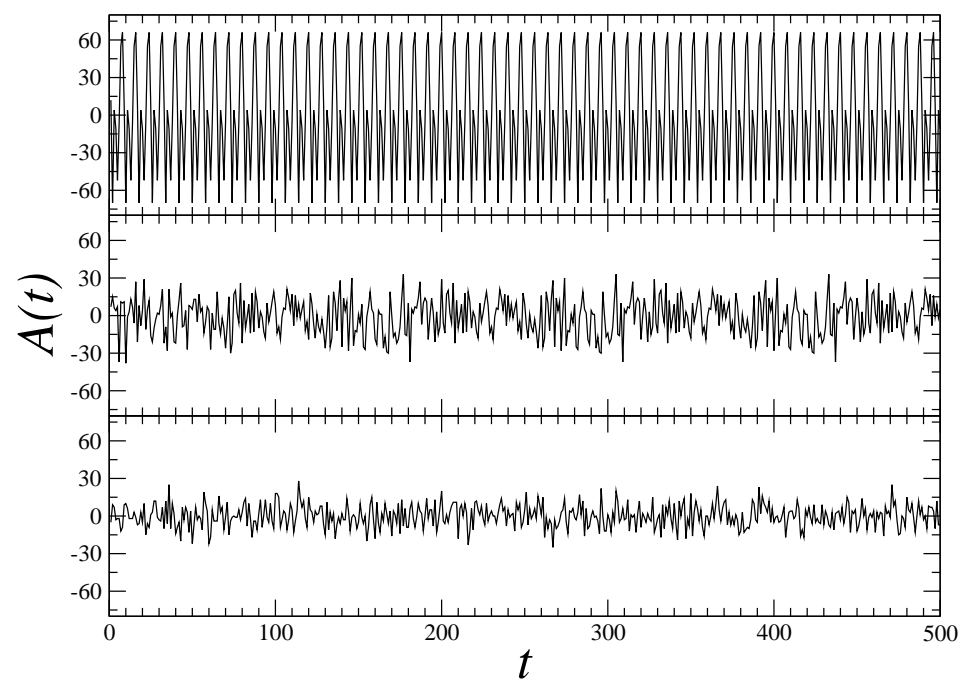

Figure 4.1: Time evolution of the attendance $A(t)$ with $g[A(t)]=A(t), 2 k+1=301$ and $n_{S}=2$. Panels correspond to $m=2,7,15$ from top to bottom. Figure taken from Moro $(2003)$.

the volatility converges to the volatility exhibited in the symmetric mixed-strategy Nash equilibrium for $\alpha \rightarrow \infty$. With a large number of players ( $\alpha$ small), overall performance is much worse; in fact, the volatility is of the order of $(2 k+1)^{2}$, so that the size of the winning group is much smaller than $k$. At intermediate values of $\alpha$, volatility is low, and it attains a minimum at $\alpha_{c}\left(n_{S}\right) \cong n_{S} / 2-0.66$ (Marsili et al., 2000). Hence, at intermediate values of $\alpha$, players are able to coordinate their actions and perform better collectively than under the symmetric mixed-strategy Nash equilibrium. This means that players can exploit the available information to predict future market movements so that the aggregate welfare loss $\sigma^{2}$ is reduced relative to the symmetric mixed-strategy Nash equilibrium. Note that this is not the result of some form of cooperative behavior of the players: agents are selfishly maximizing their own return, and in doing that, they come closer to global efficiency.

However, coordination is not complete under the current learning model. In the socially efficient outcome, players would play according to one of the pure-strategy Nash equilibria of the game, and the minority would consist of $k$ players. In that case, almost half of the players are in the minority, and $\sigma^{2} /(2 k+1)=1 /(2 k+1)$. Players come close to this optimum at $\alpha=\alpha_{c}$, although they never reach it. For smaller values of $\alpha$, performance is much worse than under this optimum, while for large values of $\alpha$, aggregate payoffs are close to those of the symmetric mixed-strategy Nash equilibria (see Figure 4.2). By 


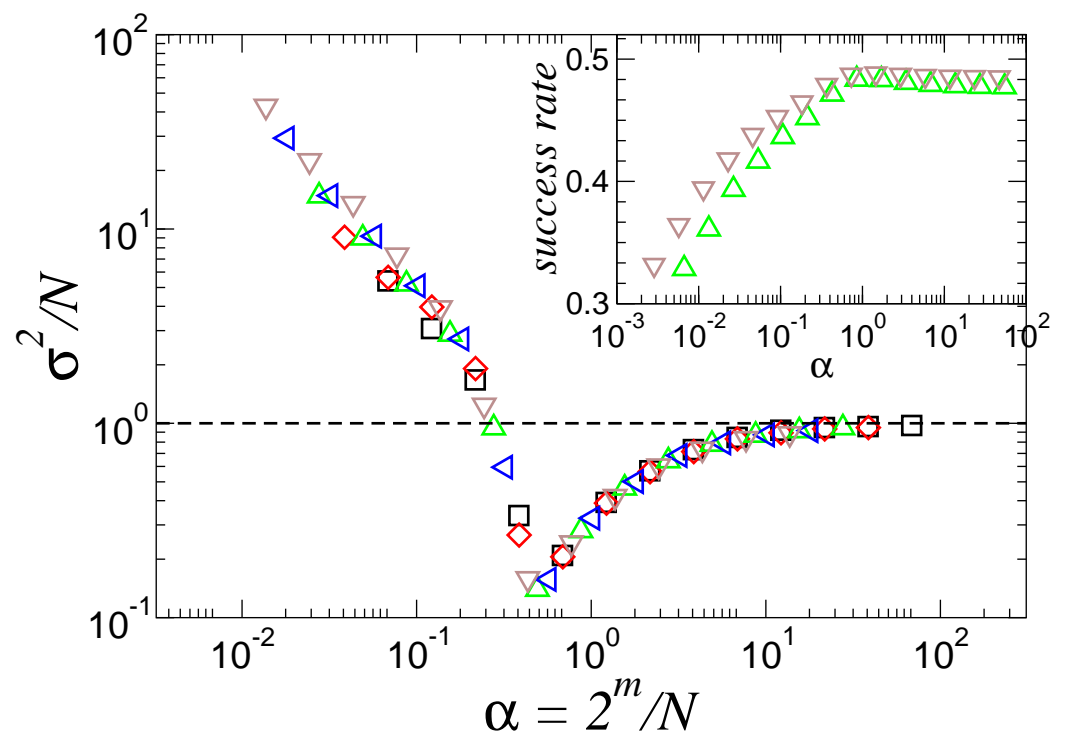

Figure 4.2: Volatility as a function of the order parameter $\alpha$ for $n_{S}=2$ and different number of players $N:=2 k+1=101,201,301,501,701(\square, \diamond, \triangle, \triangleleft, \nabla$, respectively). The critical value $\alpha_{c}$ is the value of $\alpha$ for which the volatility is at a minimum. Inset: Agent's mean success rate as function of $\alpha$. Figure taken from Moro (2003).

contrast, when players do take the effect of their own action on the aggregate outcome into account, play converges to one of the pure-strategy Nash equilibria of the game so that coordination is complete (Challet et al., 2000b; Marsili et al., 2000; Marsili and Challet, 2001; De Martino and Marsili, 2001) 12

Strikingly, global efficiency is enhanced for certain values of $\alpha$ when players do not always choose the response mode $s$ with the highest number of virtual points, i.e. when $\beta<\infty$ in Equation (3.1). It can be shown that for $\alpha<\alpha_{c}$ (the socially inefficient regime), volatility decreases when the noise level increases. For $\alpha>\alpha_{c}$, the value of $\beta$ does not affect the level of volatility (Cavagna et al., 1999; Challet et al., 2000a; Bottazzi et al., 2001; Marsili, 2004). This result is not so surprising, however, if one recalls that in the minority game learning model, rational players herd in the socially inefficient regime $\left(\alpha<\alpha_{c}\right)$. When $\alpha<\alpha_{c}$, there are few response modes relative to the number of players. In that case, players have to crowd at a limited number of response modes, leading to a large number of players choosing the same alternative (see Section 4.3). Setting $\beta<\infty$ is equivalent to slowing down the updating of virtual scores for response modes more slowly.

\footnotetext{
${ }^{12}$ Also, Kets and Voorneveld (2007) show that most standard learning processes such as the replicator dynamic converge to the pure-strategy Nash equilibria of the game.
} 
A finite $\beta$ therefore acts as a brake against overreaction (Bottazzi et al., 2001) 13

To summarize, the minority game learning model is characterized by competition and coordination. Agents compete in trying to exploit asymmetries in the games outcome, but at the same time, they try to reduce volatility, as volatility harms all players. Hence, there is a tension between competition and coordination. These two are intimately linked in the minority game learning model, as are information and efficiency. We discuss these issues in more detail in the next section.

\subsection{Information and efficiency}

As discussed in the previous section, players seem to be able to coordinate reasonably well for some parameter configurations. The only way players can interact is through the virtual scores of their response modes, implying that there is some information in these values (Challet and Zhang, 1998). This observation led some authors to study the information contained in the history of play. The information content of the history of play, or the degree of predictability can be measured by (Challet and Marsili, 1999)

$$
H:=\frac{1}{2^{m}} \sum_{\nu=1}^{2^{m}}\left\langle A(t+1) \mid h_{m}(t)=\nu\right\rangle^{2},
$$

where the time average of $A(t+1)$ is conditioned to the requirement that the last $m$ winning groups are given by $h_{m}(t)$. If $A(t+1)$ and $h_{m}(t)$ are independent, then $H=0$. Loosely speaking, $H$ measures the information in the time series of $A(t)$. If $H>0$, then the signal $A(t)$ contains information. It can be shown that players in the minority game learning model minimize the degree of predictability (Challet et al., 2004). Depending on the value of $\alpha$, they are more or less successful in doing that. At $\alpha_{c}$, the system changes from an informationally efficient and socially inefficient phase $\left(H=0, \sigma^{2}\right.$ large $)$ to an information-rich and socially efficient phase $\left(H>0, \sigma^{2}\right.$ small). In the informationally efficient phase, players do worse than players playing according to the symmetric mixedstrategy Nash-equilibrium. By contrast, in the information rich phase, players manage to coordinate and do better than players who play according to the symmetric mixed-strategy Nash equilibrium.

At $\alpha=\alpha_{c}$, the symmetry between the two actions is broken. In the so-called symmetric phase $\left(\alpha<\alpha_{c}\right)$, both actions are equivalent. Both actions are taken by the players with

\footnotetext{
${ }^{13}$ This result is reminiscent of the findings of Goeree et al. (2004) who show that payoff-dependent noise in the decision process is able to break the cascades that would result otherwise in a social learning model.
} 


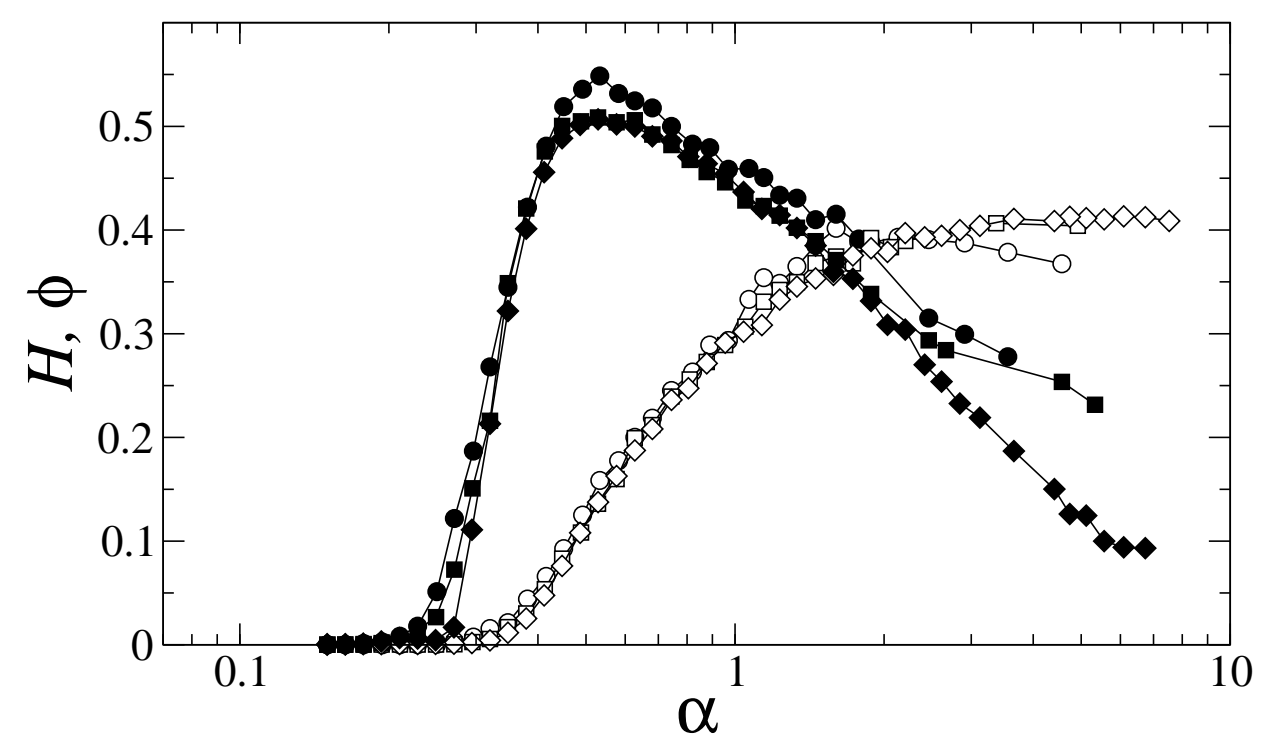

Figure 4.3: Information $H$ (open symbols) and fraction of frozen players $\phi$ (full symbols) as a function of the control parameter $\alpha=2^{m} /(2 k+1)$ for $n_{S}=2$ and $m=5,6,7$ (circles, squares and diamonds, respectively). Figure taken from Moro (2003).

equal frequency. For $\alpha>\alpha_{c}$, one of the actions is preferred, i.e. the outcome is asymmetric. An asymmetry in the game's outcome represents an opportunity that could in principle be exploited. Hence, this is just a concomitant feature of the presence or absence of information in the history of play.

As an alternative to $H$, one could also consider the fraction of frozen players (Challet and Marsili, 1999). Frozen players are players who never change their response mode in the stationary state (in the limit of $\beta \rightarrow \infty$ in Equation (3.1)). That is, these players have one response mode that outperforms all others 14 As can be seen from Figure 4.3, the fraction $\phi$ of frozen players is zero in the informationally efficient phase, while it first rises for intermediate values of $\alpha$ and then falls again when $\alpha$ goes to infinity. The intuition is that, for very small values of $\alpha$ (the informationally efficient phase), both actions are equivalent, so that there is little variation in the virtual scores of the different response modes. This means that players switch response modes easily. For very large values of $\alpha$, players behave more or less randomly, so they switch response modes frequently. Only at intermediate values of $\alpha$ the fraction of frozen players is large, as many players have a response mode that is superior to other response modes. Note that the success of a response mode depends on

\footnotetext{
${ }^{14}$ Note that this does not imply that these players take the same action always: a response mode is a function of past play, hence the actions vary with the history $h_{m}$.
} 
the response modes used by opponents: a response mode per se is not superior, it is the collective of response modes that is successful (see also Savit et al., 1999). In particular, it only pays to be predictable if others are predictable as well.

This transition between the informationally efficient and the information rich phase, or equivalently between the socially inefficient and the socially efficient phase, is central to the minority game learning model. At this transition, there is a qualitative change in collective behavior, while the principles behind the behavior of individuals remain unchanged. For all values of $\alpha$, players in the minority game learning model try to outsmart each other, but for low values of $\alpha$, they are on average less successful. In the next section, we discuss the interpretation of $\alpha$.

\subsection{Response modes and their antagonists}

The former sections have shown that the qualitative behavior of the system depends only on $\alpha=2^{m} /(2 k+1)$, not on other variables such as $n_{S}$. Moreover, for some values of this parameter, players are much more successful in coordinating behavior than for other values. What is the feature of the model underlying this behavior? We address this question in the current section. The answer to this question points to an intuitive interpretation of the model's results in terms of response modes and their antagonists.

The minority rule forces players to differentiate: if all players choose the same response mode, all will loose. Agents want to be as far apart in the space of response modes as possible. However, there are only $2^{2^{m}}$ possible response modes for $2 k+1$ players. Hence, one would expect that players succeed in differentiating if $2 k+1 \ll 2^{2^{m}}$, while they behave more like a crowd when $2 k+1>2^{2^{m}}$. So, one would expect a qualitative change at $2 k+1 \sim 2^{2^{m}}$, rather than at $2 k+1 \sim 2^{m}$, as observed. The reason that the transition occurs at $2 k+1 \sim 2^{m}$ rather than at $2 k+1 \sim 2^{2^{m}}$ is that two response modes $s, s^{\prime}$ only give rise to distinctively different behavior if either they prescribe different actions for every history of play (i.e., $s$ and $s^{\prime}$ are anti-correlated) or if their predictions are uncorrelated (Challet and Zhang, 1998; Johnson et al., 1998; Hart et al., 2001). It can be shown that for every response mode $s$, the number of response modes that are anti-correlated or uncorrelated with $s$ is given by $2 \cdot 2^{m} / n_{S}$ (Challet and Zhang, 1998; Hart et al., 2001). Hence, $\alpha$ is proportional to the inverse of this number.

This leads us to an intuitive interpretation of the model's results in terms of the interplay between different response modes. Let $s$ be a response mode, and let $\bar{s}$ be the response mode that is anti-correlated with $s$. Suppose $N_{s}$ players use the response mode $s$ at a given 
time step, and $N_{\bar{s}}$ players use the anti-correlated response mode $\bar{s}$ at the same time step. If $N_{s} \approx N_{\bar{s}}$ for all anti-correlated pairs $(s, \bar{s})$ of response modes, then the actions of players using these response modes effectively cancel and the volatility will be small.

Hence, it would be optimal if the group of players that use a certain response mode is of about the same size as the group that uses the "antagonistic" response mode. However, this is not always possible, as the dimension of the space of response modes is fixed by the parameter $m$. Hence, players can only be "far apart" in terms of response modes if the number of players is not too large relative to the dimension of the response mode space. For a given number of players, players cannot differentiate if $m$ is small, as the space of response modes is too crowded in that case. The players display herding behavior: for a pair of anti-correlated response modes $(s, \bar{s})$, almost all players herd at one of them, with very few players choosing the other. Hence, the actions of the players choosing a given response mode do not cancel those of the players using its antagonist, so that $\sigma^{2}$ will be large. For somewhat larger $m$ (for a fixed number of players), players can differentiate, and the actions of players effectively cancel. Hence, the system is quite successful collectively at intermediate values of $\alpha$, although the minority rule prevents the system from attaining full efficiency, i.e., not all players can be on the minority side. For a given $h_{m}$, the response modes of most players are uncorrelated, but a small share of players uses response modes that are mutually anti-correlated. This coordinated avoidance is beneficial for everybody, as it helps to get a more even division of players over both alternatives (Zhang, 1998).

Now, for very large $m$ at a fixed number of players, the number of players using a given response mode will only be small, so that players act more or less independently (Moro, 2003). However, the system still performs better than players who play the symmetric mixed-strategy Nash equilibrium would, as there always exists pairs of players that follow anti-correlated response modes, so that the players' actions are never truly independent and $\sigma^{2} /(2 k+1)$ is smaller than 1 , the value of $\sigma^{2} /(2 k+1)$ under the symmetric mixed-strategy Nash equilibrium (Challet and Zhang, 1998).

\section{Comparison to experimental results}

In this section, we discuss some experiments on the minority game and related congestion games. In addition to the minority game, we focus on market entry games and route-choice games. First, we briefly introduce these two classes of games. We then present some experimental results, and discuss whether and how the learning model proposed in the minority game literature could explain these results. 
The market entry game (Selten and Güth, 1982) has been studied extensively in economics 15 In a market entry game, $N \in \mathbb{N}$ players must decide independently and simultaneously to enter a market with a fixed capacity $c<N$ or to stay out. Players who enter the market receive a payoff that decreases in the number of entrants. The payoff of players who stay out of the market is commonly taken to be constant. The game generally has a large number of Nash equilibria, both in pure and in mixed strategies. Depending on the exact form of the payoff function, there may even be a continuum of equilibria. Pure-strategy Nash equilibria may be payoff-symmetric or payoff-asymmetric, and strict or non-strict, depending on the choice of parameters. For the payoff functions commonly studied, the number of entrants is between $c-1$ and $c$ in equilibrium (Erev and Rapoport, 1998; Duffy and Hopkins, 2005). An important difference between the market entry game and the minority game is that in the latter game, congestion effects are symmetric, while in the former game, players can choose between a safe option with guaranteed payoffs staying out - and entering, the payoffs of which depends on the number of other players that enter.

As the market entry game is a congestion game, the fictitious play process converges in beliefs to one of the Nash equilibria of the game (Monderer and Shapley, 1996a). Duffy and Hopkins (2005) show that the evolutionary replicator dynamic converge to one of its rest points, and that the mixed-strategy Nash equilibria of the game are unstable under the dynamic. They also show that under standard reinforcement learning (Roth and Erev, 1995), the learning process converges with probability one to one of the pure-strategy Nash equilibria of the game (when $c \notin \mathbb{N}$ ). Under hypothetical reinforcement learning, where also the propensities of strategies not used are updated, the learning process converges with probability one to one of the (logit) perturbed equilibria corresponding to the pure-strategy Nash equilibria of the game for $c \notin \mathbb{N}$ (Duffy and Hopkins, 2005). 16

Route-choice games are closer to the minority game in that there is no safe option. In a route-choice game, players choose between two or more roads. The payoffs of choosing one of these roads falls in the number of other players who have chosen that road. Roads may differ in terms of capacity. In equilibrium, players divide themselves over the roads in such a way that traveling times and hence payoffs are equalized. These games have been studied

\footnotetext{
${ }^{15}$ See e.g. Duffy and Hopkins (2005), Kahneman (1988), Rapoport (1995), Sundali et al. (1995), Erev and Rapoport (1998), Rapoport et al. (1998, 2000).

${ }^{16}$ The perturbed equilibria are the logit quantal response equilibria (McKelvey and Palfrey, 1995) of the game.
} 
experimentally by a number of authors 17 An important difference with the minority game is that the pure-strategy Nash equilibria of the route-choice game are payoff-symmetric. Moreover, these Nash equilibria are strict, unlike in the minority game. It is easy to see that the fictitious play process converges in beliefs to one of the Nash equilibria of the game (Monderer and Shapley, 1996a). No other analytic results are available on the behavior of different learning processes in this type of games; however, given the similarities with market entry games and the minority game, we may expect learning processes to behave similarly in these games.

The minority game has been discussed in detail in Section 2. Kets and Voorneveld (2007) study the predictions of different learning models for the minority game. They show that the collection Nash equilibria with at most one mixer is asymptotically stable under the multi-population replicator dynamic, while other stationary states of the replicator dynamic are not Lyapunov stable (e.g. Weibull, 1995). Finally, as in all congestion games, the fictitious play process converges in beliefs to one of the Nash equilibria of the game.

We now discuss some experimental results on market entry games, route-choice games and the minority game, and whether, and how, these results can be explained by the minority game learning model. A robust finding in experiments on these games is that subjects quickly achieve a "magical" degree of coordination. However, individual players generally do not play equilibrium strategies. For instance, while Erev and Rapoport (1998) find that the number of entrants in a market entry game rapidly converges to the equilibrium value, they also observe large between- and within-subject variability, which does not diminish with experience. This is a common finding in experiments on market entry games (Ochs, 1999, p. 169) 18 Similarly, in experiments on a route-choice game, Selten et al. (2007) observe that the mean number of drivers on the different roads is very close to the equilibrium number, while large fluctuations persist until the end of the session. Similar experimental results have been reported for the minority game (Chmura and Pitz, 2004; Platkowski and Ramsza, 2003; Bottazzi and Devetag, 2007). In all cases, the hypothesis that fluctuations can be explained by a symmetric mixed-strategy Nash strategy equilibrium of the game can be rejected. These results cannot be explained with standard learning or evolutionary models, as these models typically predict convergence to the purestrategy Nash equilibria of such games (Duffy and Hopkins, 2005; Kets and Voorneveld,

\footnotetext{
${ }^{17}$ See e.g. Iida et al. (1992), Helbing et al. (2005), and Selten et al. (2007).

${ }^{18}$ An exception is Duffy and Hopkins (2005) who find that subjects coordinate on one of the pure Nash equilibria of the market entry game after a large number of rounds when they are given feedback on others' choices.
} 
2007). However, as discussed in Section 4.1, the minority game learning model predicts precisely that average behavior will be close to the equilibrium prediction, while fluctuations will persist.

Some authors attempt to reconcile aggregate "equilibrium" behavior in experiments with individual non-equilibrium play by conjecturing that subjects may use counteracting behavioral rules 19 For instance, Selten et al. (2007) report that some subjects revise their choice if the road of their choice turned out to be congested, while other players stick with their choice in that case, as they expect others to switch. Also Bottazzi and Devetag (2007) find that there is considerable heterogeneity in players' behavior in their experiments on the minority game. They show that it is not the heterogeneity per se which determines the players' success in coordinating, rather, it is the interaction between these different behavioral rules that players can successfully coordinate on choosing different actions. These findings are in line with the predictions of the minority game learning model that response modes and their antagonists coevolve in such a way that their actions effectively cancel out, thus reconciling aggregate equilibrium behavior and individual non-equilibrium play.

However, it is not fully clear which behavioral rules subjects employ. For instance, Selten et al. (2007) are unable to classify $42 \%$ of the subjects in terms of the behavioral rules they use in their route-choice experiments. This leaves open the possibility that subjects use some response modes that may not have an intuitive interpretation and are thus not recognized by the experimenters, but that nevertheless perform well as response modes and their antagonists coevolve, as predicted by the minority game learning model (see Section 3.2 and 4.3). A systematic study of the different response modes used by experimental subjects seems needed. Indeed, Zwick and Rapoport (2002) conclude that there is a need "to re-orient research on interactive decision making to individual differences, identify patterns of behavior shared by subsets of players ..., and then attempt to account for aggregate behavior in terms of the behavior of the clusters of players that form these aggregates".

Finally, the effect of information on players' behavior in such games remains a puzzle. Two dimensions of information have been investigated in the experimental literature. Firstly, it has been studied how behavior depends on the information given on other players' choices. Players can be provided with information only on the payoff rule and aggregate behavior in the past rounds or may be informed additionally of the individual choices of all

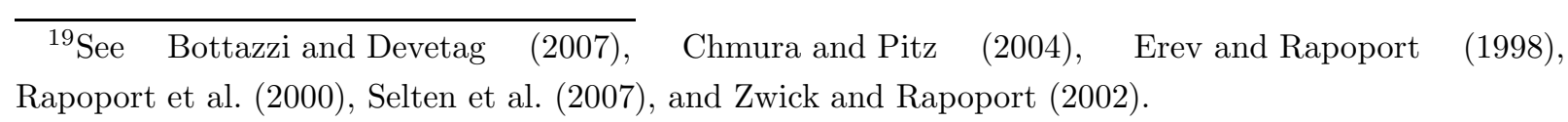


other players. If players learn e.g. according to the standard reinforcement learning model of Roth and Erev (1995), hypothetical reinforcement (Duffy and Hopkins, 2005), the minority game learning model, or if the learning process can be described by the replicator dynamic, this should not affect results.

However, in many experimental studies, behavior differs qualitatively depending on the information players have. Duffy and Hopkins (2005) reports that behavior becomes less random when players are provided with information on the individual choices of other players: the hypothesis of randomizing behavior can be rejected for a larger share of the players, and subjects seem to display some inertia in their behavior. However, this may be due to the fact that the additional information given to players allows them to play complicated repeated-game strategies: players may signal their commitment to a certain action. While for the market entry game, such a signalling strategy pays off, this is not the case in the minority game 20 Also, one can imagine that feelings like regret or envy play a larger role in the market entry game (Erev and Rapoport, 1998). In that sense, experiments on the minority game provide a cleaner test of learning theory. Nevertheless, Bottazzi and Devetag (2007) find that providing players with additional information on their opponents' play makes that players switch less often between different actions. In the treatment with full information on individual players' actions, players tend to stick more often to their last period's action, especially when this action was the minority action. Combined with some heterogeneity in players' beliefs, this inertia and "reinforcement" effect partly explains players' success at coordinating in the minority game. However, Bottazzi and Devetag show that inertia, reinforcement, and heterogeneity alone are not sufficient: players' strategies also coevolve, or self-organize to improve aggregate payoffs, as predicted by the minority game learning model.

A second dimension of information that has been studied in the literature refers to the salience of information on the recent history of play. Bottazzi and Devetag (2007) provide players with a string of past outcomes of varying length. When players are provided with information on play in more rounds than just the previous one, aggregate efficiency is

\footnotetext{
${ }^{20}$ For instance, suppose that $k$ players commit to action $a=-1$, and $k$ players commit to action $a=+1$. The remaining player will not be deterred from choosing either of those actions by the commitment of other players, nor does the commitment of these players guarantee them a positive payoff. A repeated-game strategy that does pay off in the minority game is one in which players "take turns": players alternately choose each of the two actions in such a way that each player is in the minority roughly half of the time. Indeed, Helbing et al. (2005) find some evidence of such behavior in their experiments on route-choice games with small groups, but it is unlikely that players will be able to successfully play according to such a repeated-game equilibrium when the number of players is large.
} 
significantly improved. They find that providing players with a string of greater length allows players to correlate their behavior over a longer time period: when players are provided with the outcome of the previous round, there is only a significant relation between present and past choices for the first two time lags, whereas such a relation hold for up to three time lags when more information is provided. Notably, in a treatment where players are provided with a string of intermediate length and the degree of aggregate efficiency is highest, play is characterized by a substantial lack of short-range correlations between current and past actions: players seem to exploit the additional information to improve their payoffs.

All together, these experimental studies give some support to the learning model proposed in the minority game literature. However, the question how information influences play in congestion games has still not been satisfactorily answered. It would be interesting to compare players' behavior under different informational treatments in different congestion games. While most learning models make similar predictions for the different congestion games discussed here, intuitively, one would expect that information will play a different role in these games, as emotions like envy and regret will be more important in some games than in others, and also the scope for repeated-game strategies differs across games. Such a systematic comparison would allow one to better separate the learning effects from possible repeated-game and behavioral effects.

\section{Conclusions}

In this paper, we have given a critical account of the learning model proposed in the learning model proposed in the minority game literature, and related it to standard learning and evolutionary models in economics, showing that it shares quite a few features with these models. Still, the predictions of this learning model are markedly different from the predictions from other models. However, these predictions are in line with some experimental results on the minority game and related games, which cannot be explained by other models.

However, our understanding of learning in such games is far from complete. For instance, the effect of feedback on play is unclear. An interesting direction for further research would be to systematically vary players' information in experiments on different congestion games such as the minority game and the market entry game, and to compare play under the different information treatments and across games. While most learning models provide similar predictions for these games, intuitively, one would expect that information 
may have different effect in these games, as in some games, repeated-game strategies or emotions may play a larger role than in others. Such an experiment may help shed light on the question which learning model is appropriate in such games.

\section{References}

Arthur, W. B. (1994). Inductive reasoning and bounded rationality. American Economic Review 84, 406-411.

Aumann, R. (1997). Rationality and bounded rationality. In S. Hart and A. Mas-Colell (Eds.), Cooperation: Game-Theoretic Approaches, Volume 155 of NATO ASI Serie F. Berlin: Springer.

Blonski, M. (1999). Anonymous games with binary actions. Games and Economic Behavior 28, 171-180.

Bottazzi, G. and G. Devetag (2004). Coordination and self-organization in minority games: Experimental evidence. In M. Gallegati, A. Kirman, and M. Marsili (Eds.), The Complex Dynamics of Economic Interaction: Essays in Economics and Econophysics, Volume 521 of Lecture Notes in Economics and Mathematical Systems. Berlin: Springer-Verlag.

Bottazzi, G. and G. Devetag (2007). Competition and coordination in experimental minority games. Journal of Evolutionary Economics 17, 241 - 275.

Bottazzi, G., G. Devetag, and G. Dosi (2001). Adaptive learning and emergent coordination in minority games. LEM Working Paper Series, Sant'Anna School of Advanced Studies 1999/24.

Camerer, C. and E. Fehr (2006). When does "econonomic man" dominate behavior? Science 311, $47-52$.

Camerer, C. and T.-H. Ho (1999). Experience-weighted attraction learning in normal form games. Econometrica 67, 827-874.

Camerer, C., T.-H. Ho, and J.-K. Chong (2004). A cognitive hierarchy model of one-shot games. Quarterly Journal of Economics 119, 861-898.

Cavagna, A., J. P. Garrahan, I. Giardina, and D. Sherrington (1999). A thermal model for adaptive competition in a market. Physical Review Letters 83, 4429-4432. 
Challet, D. and M. Marsili (1999). Phase transition and symmetry breaking in the minority game. Physical Review E 60, R6271-R6274.

Challet, D., M. Marsili, and R. Zecchina (2000a). Comment on: Thermal model for adaptive competition in a market. Physical Review Letters 85, 5008.

Challet, D., M. Marsili, and R. Zecchina (2000b). Statistical mechanics of systems with heterogeneous agents: Minority games. Physical Review Letters 84, 1824-1827.

Challet, D., M. Marsili, and Y.-C. Zhang (2004). Minority Games: Interacting Agents in Financial Markets. Oxford: Oxford University Press.

Challet, D. and Y. C. Zhang (1997). Emergence of cooperation and organization in an evolutionary game. Physica A 246, 407-418.

Challet, D. and Y. C. Zhang (1998). On the minority game: Analytical and numerical studies. Physica A 256, 514-532.

Chmura, T. and T. Pitz (2004). Minority game - Experiments and simulations of traffic scenarios. Bonn Econ Discussion Papers 23/2004.

Coolen, A. (2005). The Mathematical Theory of Minority Games: Statistical Mechanics of Interacting Agents. Oxford: Oxford University Press.

De Martino, A. and M. Marsili (2001). Replica symmetry breaking in the minority game. Journal of Physics A 34, 2525-2537.

DeAngelis, D. and L. Gross (1992). Individual-Based Models and Approaches in Ecology: Populations, Communities, and Ecosystems. New York: Chapman and Hall.

Duffy, J. and E. Hopkins (2005). Learning, information, and sorting in market-entry games: theory and evidence. Games and Economic Behavior 51, 31-62.

Ellison, G. and D. Fudenberg (1993). Rules of thumb for social learning. Journal of Political Economy 101, 612 - 643.

Erev, I. and A. Rapoport (1998). Coordination, "magic," and reinforcement learning in a market entry game. Games and Economic Behavior 23, 146-175.

Erev, I. and A. Roth (1998). Predicting how people play games: Reinforcement learning in experimental games with unique mixed strategy equilibria. American Economic Review 88, 848-881. 
Fudenberg, D. and D. Levine (1998). The Theory of Learning in Games. Cambridge, MA: MIT Press.

Goeree, J. K., T. R. Palfrey, B. W. Rogers, and R. D. McKelvey (2004). Self-correcting information cascades. California Institute of Technology Social Science Working Paper 119\%. Forthcoming in The Review of Economic Studies.

Harsanyi, J. C. and R. Selten (1988). A General Theory of Equilibrium Selection in Games. Cambridge, MA: MIT Press.

Hart, M., P. Jefferies, N. F. Johnson, and P. M. Hui (2001). Crowd-anticrowd theory of the minority game. Physica A 298, 537-544.

Helbing, D., M. Schönhof, H.-U. Stark, and J. A. Holust (2005). How individuals learn to take turns: Emergence of alternating cooperation in a congestion game and the Prisoners Dilemma. Advances in Complex Systems 8, 87 - 116.

Hofbauer, J. and E. Hopkins (2005). Learning in perturbed asymmetric games. Games and Economic Behavior 52, 133-152.

Hofbauer, J. and W. H. Sandholm (2002). On the global convergence of stochastic fictitious play. Econometrica 70, 2265-2294.

Huberman, B. and R. Lukose (1997). Social dilemmas and internet congestion. Science 277, $535-537$.

Iida, Y., T. Akiyama, and T. Uchida (1992). Experimental analysis of dynamic route choice behavior. Transportation Research. Part B 26, 17 - 32 .

Johnson, N. F., S. Jarvis, R. Jonson, P. Cheung, Y. R. Kwong, and P. M. Hui (1998). Volatility and agent adaptability in a self-organizing market. Physica A 258, 230-236.

Kahneman, D. (1988). Experimental economics: A psychological perspective. In R. Tietz, W. Albers, and R. Selten (Eds.), Bounded Rational Behavior in Experimental Games and Markets, pp. 11-18. New York: Springer-Verlag.

Kets, W. and M. Voorneveld (2007). Learning in a simple congestion game. Discussion Paper, Tilburg University.

Keynes, J. M. (1936). The General Theory of Employment, Interest and Money. Macmillan Cambridge University Press. 
Kojima, F. and S. Takahashi (2004). Anti-coordination games and dynamic stability. Working Paper, Harvard University. Forthcoming in International Game Theory Review.

Li, Y., A. VanDeemen, and R. Savit (2000). The minority game with variable payoffs. Physica A 284, $461-477$.

Manuca, R., Y. Li, R. Riolo, and R. Savit (2000). The structure of adaptive competition in minority games. Physica A 282, 559-608.

Marsili, M. (2004). Statistical physics of interacting models: Minority games. mimeo.

Marsili, M. and D. Challet (2001). Trading behavior and excess volatility in toy markets. Advances in Complex Systems 4, 3-17.

Marsili, M., D. Challet, and R. Zecchina (2000). Exact solution of a modified El Farol's bar problem: Efficiency and the role of market impact. Physica A 280, 522-553.

McKelvey, R. D. and T. R. Palfrey (1995). Quantal response equilibria for normal form games. Games and Economic Behavior 10, 6-38.

Menezes, F. M. and R. Pitchford (2006). Binary games with many players. Economic Theory 28, 125-143.

Meyer, D. J., J. B. Van Huyck, R. C. Battalio, and T. R. Saving (1992). History's role in coordinating decentralized allocation decisions. Journal of Political Economy 100, $292-316$.

Monderer, D. and L. Shapley (1996a). Fictitious play property for games with identical interests. Journal of Economic Theory 68, $258-265$.

Monderer, D. and L. Shapley (1996b). Potential games. Games and Economic Behavior 14, $124-143$.

Moro, E. (2003). The minority game: An introductory guide. In E. Korutcheva and R. Cuerno (Eds.), Advances in Condensed Matter and Statistical Physics. New York: Nova Science Publishers.

Nagel, K., S. Rasmussen, and C. Barrett (1997). Network traffic as a self-organized critical phenomenon. In F. Schweitzer (Ed.), Self-organization of Complex Structures: From Individual to Collective Dynamics, pp. 579. London: Gordon and Breach. 
Ochs, J. (1990). The coordination problem in decentralized markets: An experiment. Quarterly Journal of Economics 105, 545 - 559.

Ochs, J. (1999). Coordination in market entry games. In D. Budescu, I. Erev, and R. Zwick (Eds.), Games and Human Behavior: Essays in Honor of Amnon Rapoport, pp. 143-172. Mahwah, NJ: Erlbaum.

Płatkowski, T. and M. Ramsza (2003). Playing minority game. Physica A 323, 726-734.

Rapoport, A. (1995). Individual strategies in a market-entry game. Group Decision and Negotiation 4, 117-133.

Rapoport, A., D. Seale, I. Erev, and J. Sundali (1998). Equilibrium play in large group market entry games. Management Science 44, 119-141.

Rapoport, A., D. A. Seale, and E. Winter (2000). An experimental study of coordination and learning in iterated two-market entry games. Economic Theory 16, 661 - 687.

Renault, J., S. Scarlatti, and M. Scarsini (2005). A folk theorem for minority games. Games and Economic Behavior 53, $208-230$.

Rosenthal, R. (1973). A class of games possessing pure-strategy Nash equilibria. International Journal of Game Theory 2, 65-67.

Roth, A. E. and I. Erev (1995). Learning in extensive-form games: Experimental data and simple dynamic models in the intermediate term. Games and Economic Behavior 8, $164-212$.

Sandholm, W. H. (2001). Potential games with continuous player sets. Journal of Economic Theory 97, 81-108.

Sandholm, W. H. (2007). Population Games and Evolutionary Dynamics. Cambridge, MA: MIT Press. Forthcoming.

Savit, R., R. Manuca, and R. Riolo (1999). Adaptive competition, market efficiency and phase transitions. Physical Review Letters 82, 22032206.

Schelling, T. (1960). The Strategy of Conflict. Cambridge, MA: Harvard University Press.

Selten, R. and W. Güth (1982). Equilibrium point selection in a class of market entry games. In M. Diestler, E. Fürst, and G. Schwödiauer (Eds.), Games, Economic Dynamics, and Time Series Analysis. Wien-Würzburg: Physica-Verlag. 
Selten, R., M. Schreckenberg, T. Pitz, T. Chmura, and S. Kube (2007). Commuters route choice behavior. Games and Economic Behavior 58, 394 - 406.

Stahl, D. and P. Wilson (1995). On players' models of other players: Theory and experimental evidence. Games and Economic Behavior 10, 218-254.

Sundali, J., A. Rapoport, and D. A. Seale (1995). Coordination in market entry games with symmetric players. Organizational Behavior and Human Decision Processes 64, 203-218.

Tercieux, O. and M. Voorneveld (2005). The cutting power of preparation. SSE/EFI Working Paper 583.

Weibull, J. W. (1995). Evolutionary Game Theory. Cambridge, MA: MIT Press.

Zhang, Y.-C. (1998). Modeling market mechanism with evolutionary games. Europhysics News 29, 51-54.

Zwick, R. and A. Rapoport (2002). Tacit coordination in a decentralized market entry game with fixed capacity. Experimental Economics 5, $253-272$. 